\title{
A Calibration Facility for Search Coil Magnetometers
}

\author{
E. Pulz \\ GeoForschungsZentrum Potsdam Telegrafenberg, D-14473 Potsdam, Germany
}

\begin{abstract}
A calibration facility for search coil magnetometers has been built at Niemegk Geomagnetic Observatory, GeoForschungsZentrum Potsdam. I describe here the commissioning of this facility. The usable frequency range of $10 \mathrm{mHz}$ to $100 \mathrm{kHz}$ enables calibration of all standard types of search coil magnetometer. The homogeneity is $0.1 \%$ up to a diameter of $10 \mathrm{~cm}$ and up to a length of $2.5 \mathrm{~m}$. Extensive test measurements showed broad agreement with theoretical calculations made before construction. As part of the testing, all search coil magnetometers in the GFZ geophysical equipment pool were checked. Several devices showed serious malfunctions, emphasising the value of such a calibration facility.

The calibration coil has been designed to be largely automatic, enabling non-specialists to operate it after short instruction. It can also be adapted to test other types of sensors with little effort (for example, fluxgate magnetometers). As a result, the facility should be of broad use for external institutions to calibrate their instruments.
\end{abstract}

\section{Introduction}

Varying electric currents in the ionosphere and magnetosphere generate magnetic fields which induce electric and magnetic fields within the Earth. Magnetotellurics is the study of the conductivity structure of the Earth using measurements of these magnetic and electric fields $[1,2]$. Magnetotellurics has made great progress during recent years, with complex models of Earth structure computed using modern computers. The successful calculation of such models requires very good input data. But the measurement of the magnetic field variations is not easy, because they are of small amplitude but have a very large frequency range [2]. Depending on the frequency range, different instruments are more or less suitable for the recording of geomagnetic field variations. Search coil magnetometers (SCM) are highly suitable for the frequency range from a few $\mathrm{mHz}$ up to some $\mathrm{kHz}$. These are highly complex devices with a ferromagnetic flux amplifier and with flux-feedback. However, to be used they must be transported great distances and repeatedly buried in the ground. Consequently, the mechanical demands on these instruments are great, and they can easily be damaged without such damage being clear in the measurements. Therefore, regular calibration is crucial to ensure that the geophysical measurements are not compromised. There are many search coil magnetometers in use in Germany at several institutes without easy access to calibration facilities.

To rectify this, a calibration facility for search coil magnetometers has been built at Niemegk Geomagnetic Observatory, GeoForschungsZentrum Potsdam.

\section{Specification of the Problem}

The required output of a magnetometer calibration is the frequency response function (FRF). The sensor is excited in the required frequency range, and the input excitation and output are compared, both in amplitude and in phase difference. The resulting response is a complex function of the frequency. To generate such a function, the calibration coil must clearly be large enough to contain the sensors, its magnetic field must be sufficiently homogeneous, and the coil must generate the required frequency range without phase shift. 
The following design criteria were adopted. First, it should be possible to calibrate as many different types of search coil sensors as possible. At present, search coil magnetometers are used up to $10 \mathrm{kHz}$, but to accommodate future developments, the frequency specification for the facility was chosen as $100 \mathrm{kHz}$. The field homogeneity along the calibration coil axis should be better than $0.1 \%$ to allow the possibility of calibrating other types of magnetometer, e.g. fluxgates.

\section{Design and Construction}

The size requirements led to the choice of a cylindrical geometry-the required size of a Helmholtz coil would have been too great. The required homogeneity is easily achievable with a cylindrical coil, but reaching the desired resonant frequency is much more difficult. To have a high resonance frequency, the self-capacitance of the calibration coil has to be as small as possible. Therefore, we used the following technique for our calibration coil: coils have a smaller capacitance if their windings are split between separate chambers. We proposed to have one winding per chamber. The question was then, how large should be the distance between the chambers?

We decided in favour of a diameter of windings of $30 \mathrm{~cm}$. The magnetic flux density $\mathrm{B}$ was calculated in the direction of the coil axis for distances between windings (chambers) of $5 \mathrm{~cm}$, $7 \mathrm{~cm}, 8 \mathrm{~cm}$ and $9 \mathrm{~cm}$. The resulting homogeneity at the centre of the coil is given in Fig. 1 . Then the resonance frequency was estimated for the different distances. There must be a phase shift near zero at $100 \mathrm{kHz}$.

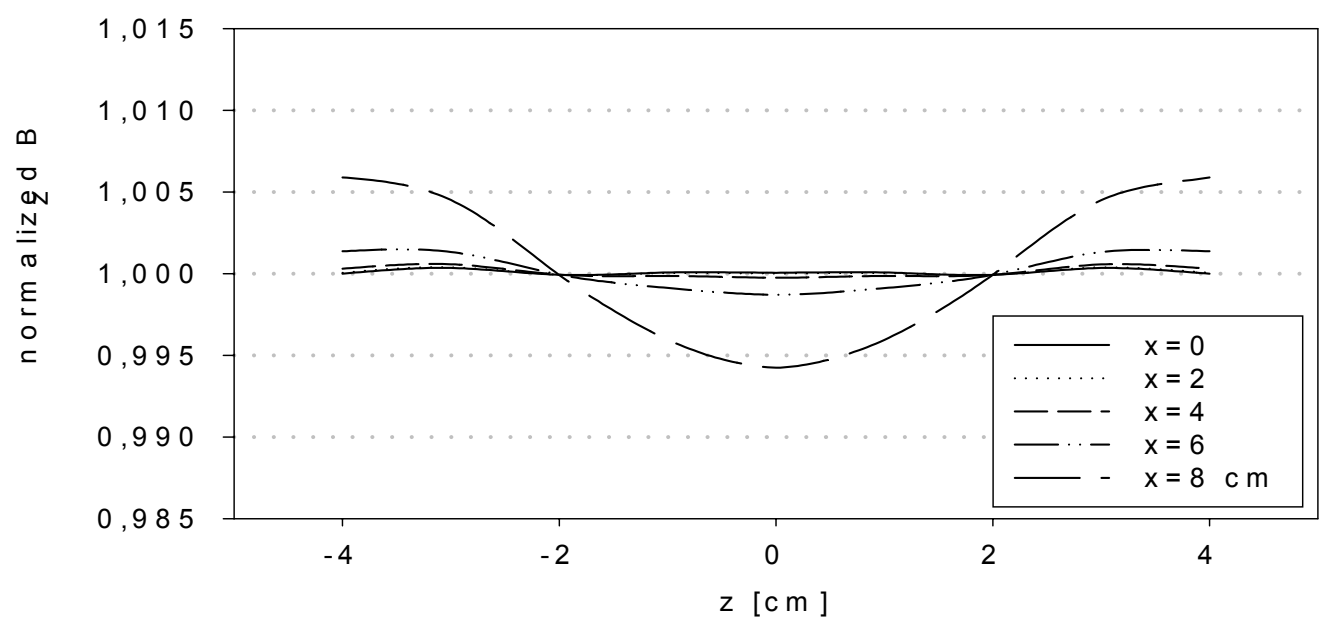

Fig. 1 Calculated magnetic flux density, z-component distance between windings $8 \mathrm{~cm}, d=30 \mathrm{~cm}$

We decided on a distance of $8 \mathrm{~cm}$ between windings, despite the risk of a phase shift at $100 \mathrm{kHz}$, because the estimated resonance frequency is $660 \mathrm{kHz}$. A small experiment to test our results was carried out, and showed that the estimated resonance frequency was very pessimistic.

The basic structure is provided by polyethylene sewage-pipe, with inner diameter $26 \mathrm{~cm}$, and wall thickness $1 \mathrm{~cm} .54$ PVC-rings (diameter $30 \mathrm{~cm}$ ) were manufactured and pushed onto the pipe, with 3 separators inserted between each pair of rings, to fix exactly the distance between rings. The connection wires are in one of these separators. The connections between the windings and the return wire (that closes the circuit) are placed anti-parallel to minimise the possible stray field. 
The coil is $4.50 \mathrm{~m}$ long. The distance between the windings was reduced continuously to the edge to improve the homogeneity at the ends (Fig. 2).

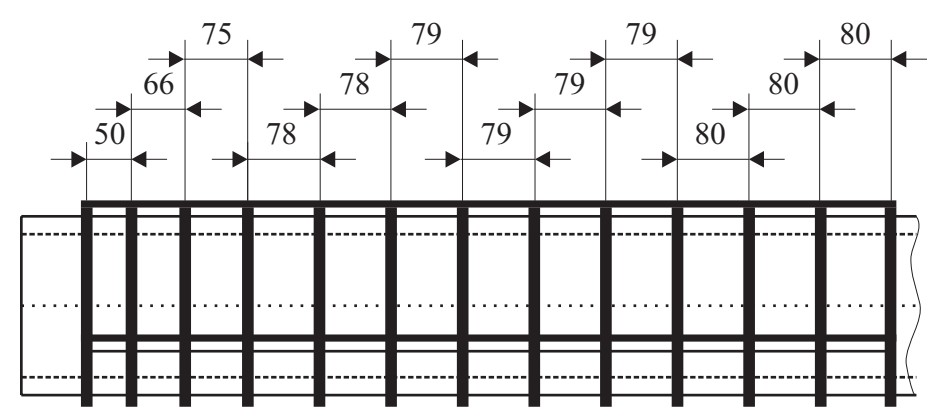

Fig. 2 Calibration coil

The coil is mounted on a plastic support, which in turn is mounted permanently on a long stone pillar in a large wooden house, the observatory magnetic laboratory. The search coil under test is pushed into the calibration coil using a specially constructed sled. Fig. 3 shoes the calibration coil before installation and Fig. 4 after installation

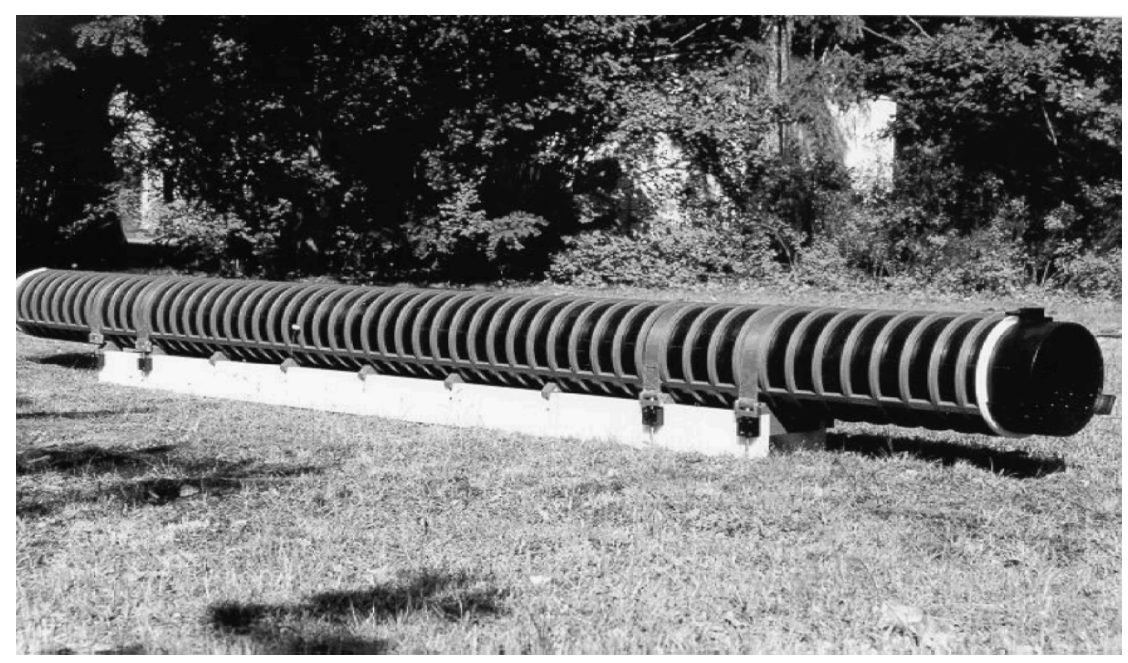

Fig. 3 Calibration coil before installation

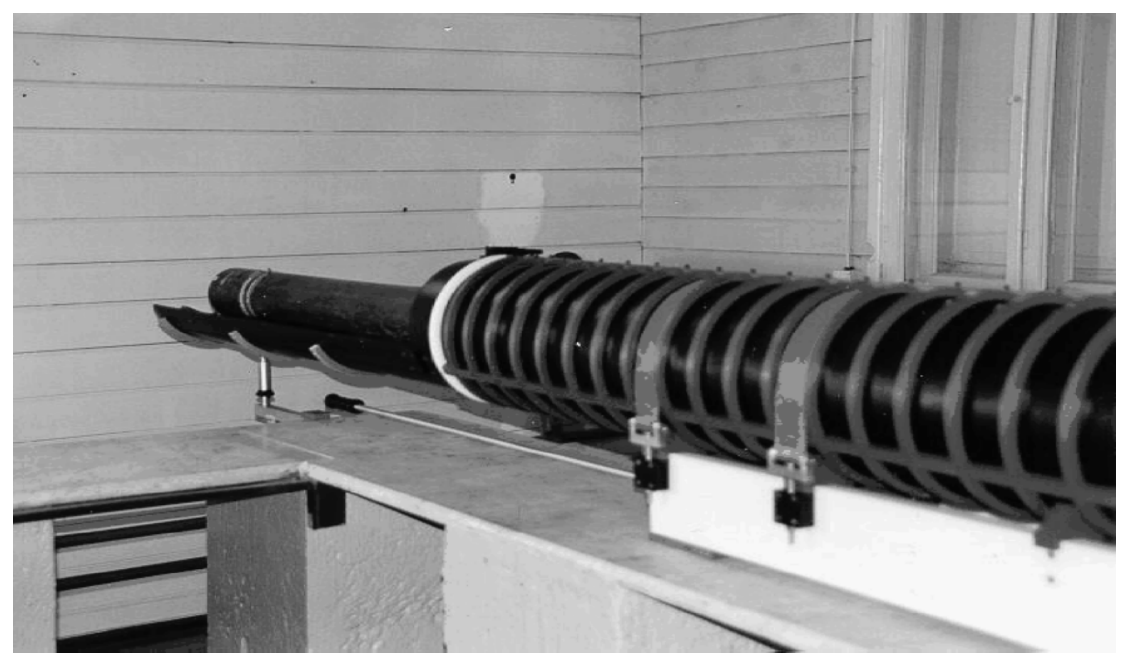

Fig. 4 Calibration coil together with search coil sensor at the sled 


\section{Measurements with the completed coil and comparison to the estimated coil parameters}

After installation, the electrical parameters of the calibration coil were determined. For results of the comparison see Table 1 . As expected the estimated and measured inductance agree very well. In contrast we found that the measured capacitance is five times less than the estimated capacitance. These values leads to a resonance frequency of $1.6 \mathrm{MHz}$. The calibration coil can therefore be used without restrictions for calibrations up to $100 \mathrm{kHz}$.

\begin{tabular}{|c|c|c|c|c|c|}
\hline & Resistance & Inductance & Capacitance & $\begin{array}{c}\text { Resonance } \\
\text { frequency }\end{array}$ & $\begin{array}{c}\text { Scale } \\
\text { factor }\end{array}$ \\
\hline calculated & & $100 \mu \mathrm{H}$ & $500 \mathrm{pF}$ & $660 \mathrm{kHz}$ & $15.65 \mathrm{nT} / \mathrm{mA}$ \\
\hline measured & $\begin{array}{c}4.6 \Omega \\
\text { at } 15.5^{\circ} \mathrm{C}\end{array}$ & $109 \mu \mathrm{H}$ & $90 \mathrm{pF}$ & $1.6 \mathrm{MHz}$ & $\begin{array}{c}15.6 \pm 0.1 \\
\mathrm{nT} / \mathrm{mA}\end{array}$ \\
\hline
\end{tabular}

Table 1 Comparison of the electrical parameters

The magnetic flux density was determined in two ways: first the Bartington MAG03 sensor was used, and secondly a small self-made air-coil was used. For both a lock-in amplifier was applied to eliminate the natural variation of magnetic field. Only a part of the measurement results are shown in Fig. 5. It shows the magnetic field inside the coil along the cylinder axis at $\mathrm{x}=0 \mathrm{~cm}, 2 \mathrm{~cm}$, and $4 \mathrm{~cm}$ over and under the axis. Further measurements were carried out at $\mathrm{x}= \pm 6 \mathrm{~cm}$ und $\mathrm{x}= \pm 8 \mathrm{~cm}$, measured every $1 \mathrm{~cm}$ because of a sinusoidal shape. It was tested for $\bar{z}=0 \mathrm{~cm}$, and $\bar{z}= \pm 75 \mathrm{~cm}$. The results do not indicate any construction defects. The homogeneity is $0.1 \%$ up to the diameter of $10 \mathrm{~cm}$ and up to a length of $2.50 \mathrm{~m}$.

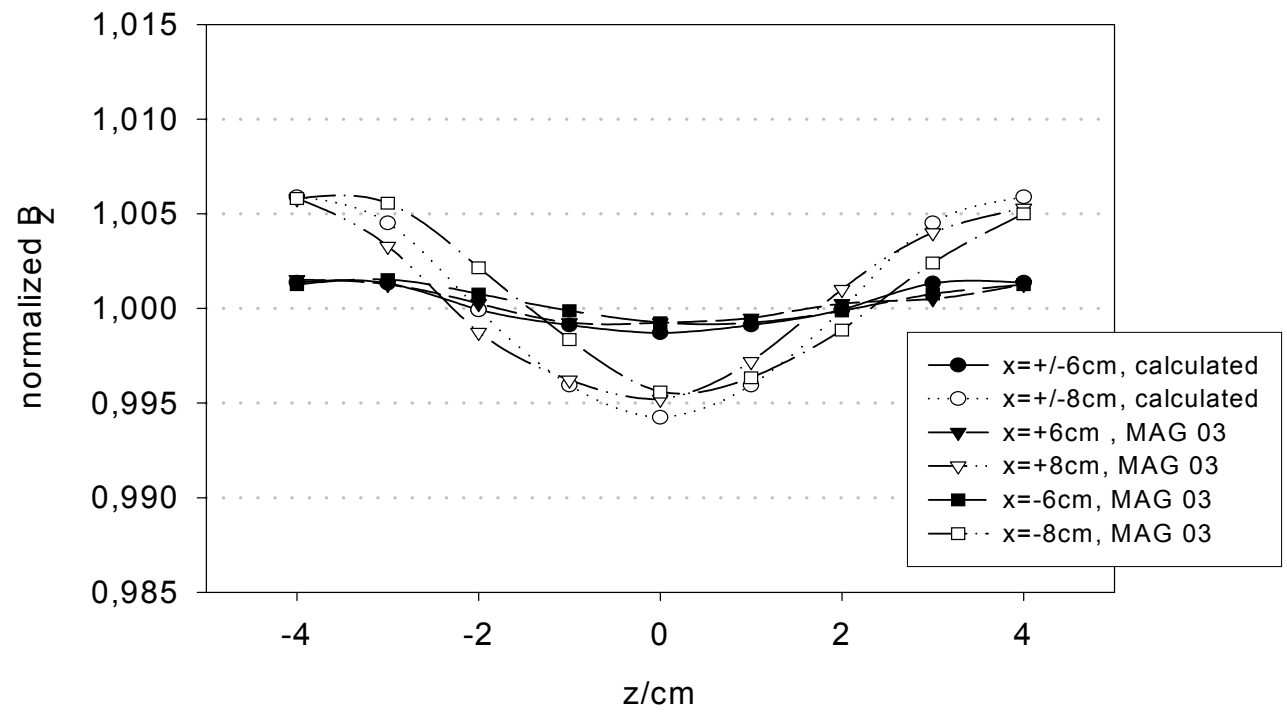

Fig. 5 Measured /MAG03) z-component

The search coil sensor is an active system containing a ferromagnetic flux amplifier. This left open the possibility of interaction between search coil and calibration coil. However, tests with SCM METRONIX sensor [3] in site gave identical results to those reported above; therefore any such interaction is so small as to not affect the calibration. 
These results demonstrate that the new calibration coil satisfies the requirements for calibrations with respect to the homogeneity, and the frequency range up to $100 \mathrm{kHz}$.

\section{The calibration facility}

To perform the calibrations, the SR785 spectrum analyser is used. This has an internal signal source and two signal inputs, and allows measurements up to $100 \mathrm{kHz}$. The measurement arrangement is shown in Fig.6. The shunt is selected for AC application. The final output is a complete picture/ data set of the FRF, both the frequency dependence of amplitude and phase:

$$
F R F=\frac{<F F T 2^{*}>\cdot<F F T 2>}{<F F T 1^{*}>\cdot<F F T 1>}
$$

where FFT denotes the Fast Fourier Transformation. The star indicates the complex conjugate value, and the number refers to the input number. The analyser allows the control of the AC level at input 1 by controlling the amplitude of the internal signal source. The calibration coil current is therefore constant even for temperature changes of the calibration coil. This option is very useful, allowing easy determination of the amplitude of the FRF in absolute units. The parameters, frequency, amplitude, and the number of the test points can be easily selected, allowing general users to carry out the calibrations after short instruction. The duration of the measurement varies according to the selected frequency range and the number of the test points. After completion of the measurement the result is graphically represented (phase and amplitude in dependence of the frequency) on the screen of the analyser. It is further possible to make a hardcopy, and the data can be saved on a diskette for further processing.

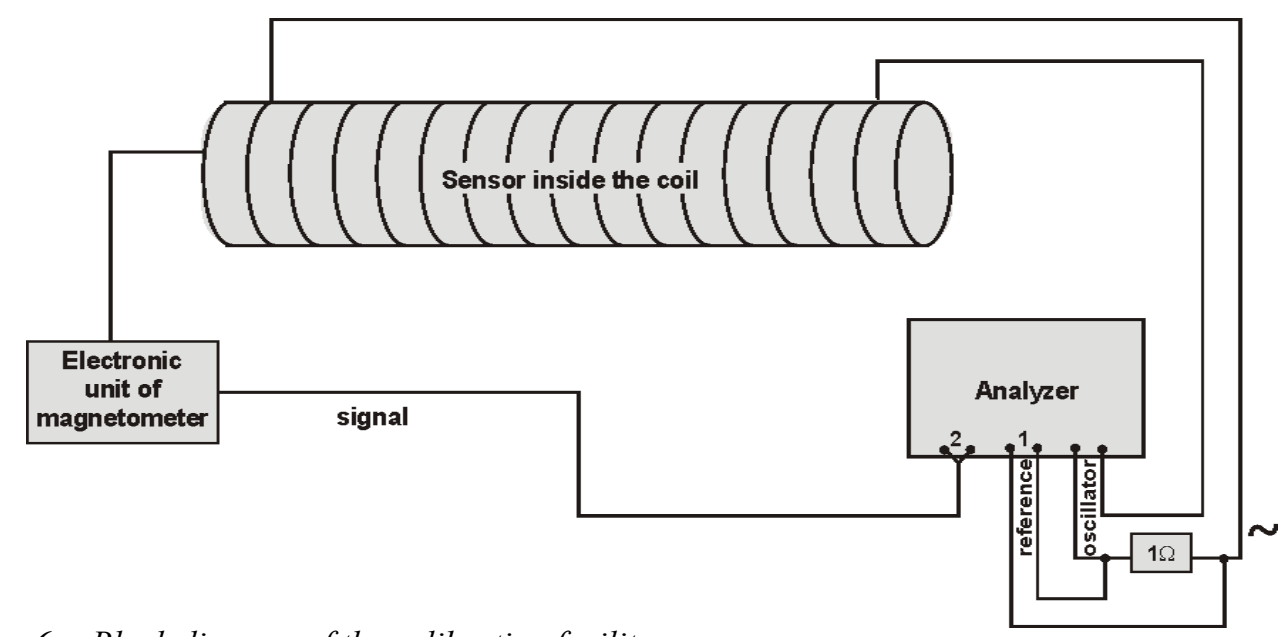

Fig. 6 Block diagram of the calibration facility

\section{Results of calibrating measurements}

Examples of calibrating measurements of METRONIX coils [3] of the GFZ equipment pool are shown as Fig. 7. These sensors can operate in both a low-frequency (LF) mode and a highfrequency (HF) mode. Correspondingly, two calibrating measurements must be carried out per device, in the LF mode of $0.1 \mathrm{~Hz}$ to $500 \mathrm{~Hz}$ and in the $\mathrm{HF}$ mode of $1 \mathrm{~Hz}$ to $10 \mathrm{kHz}$. While the LF measurement lasts for about 2 hours, the HF measurement takes only 30 minutes. The Frequency Response Function of the magnetometer No.46 is an example of an fully 
operational sensor. The peaks at $\mathrm{f}=50 \mathrm{~Hz}$ and $\mathrm{f}=16.66 \mathrm{~Hz}$ are not a malfunction of magnetometers, but are superimposed interference levels that are caused by the mains power supply and the electrified railway. The magnetometer No.35 in the LF mode, and the magnetometer No.45 in the HF mode both show significant deviations from the required FRF. It is highly unlikely that such a problem would be recognised during a field survey, leading to the collection of useless or even worse misleading results. This confirms both the necessity of calibrations and the functionality of our system. For more information look at [4].
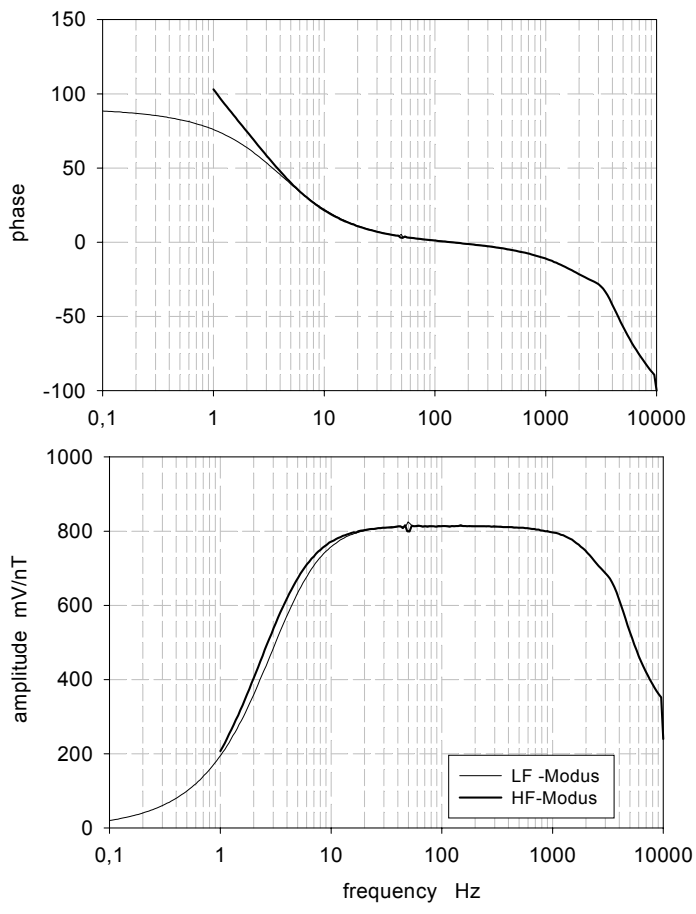
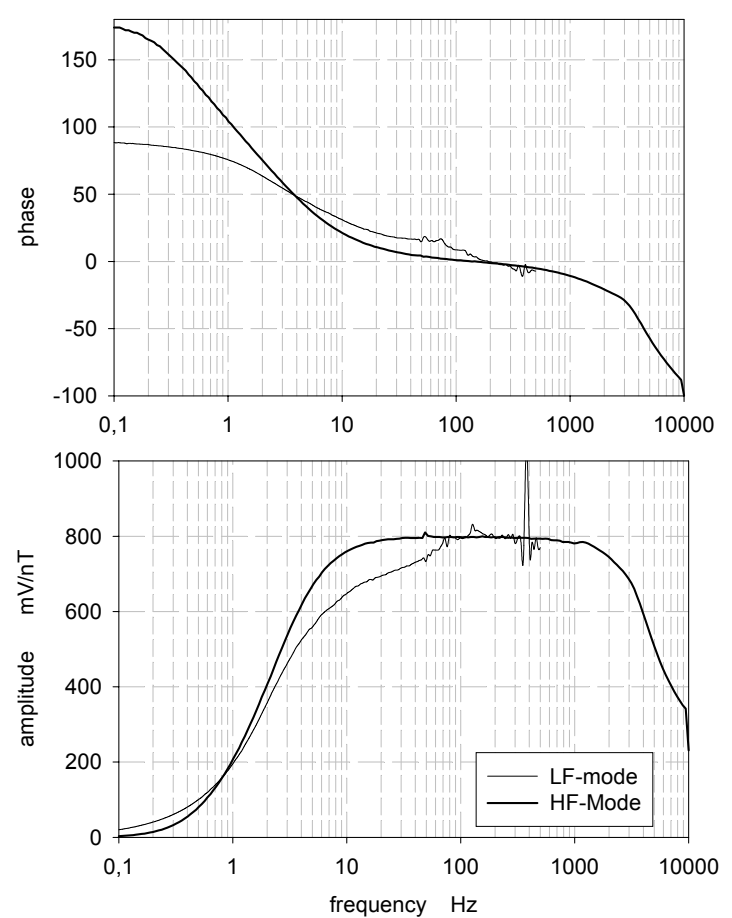
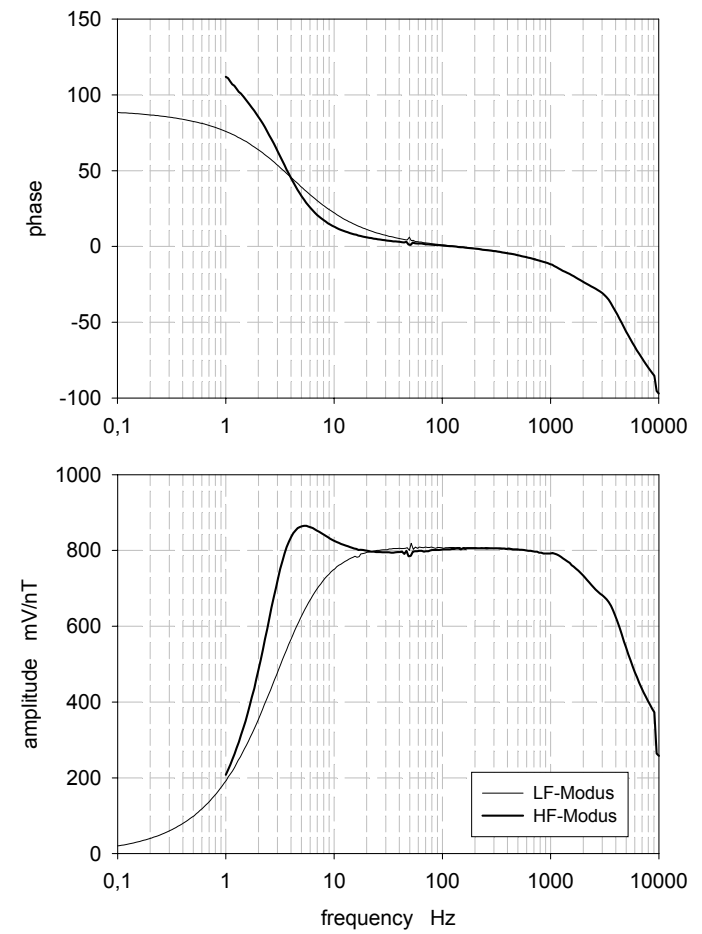

Fig. 7 Frequency Response Function of Coil No. 46, 35 and 45 (correctly operating and damaged search coil magnetometers) 


\section{Acknowledgements}

I thank V. Haak, R. Holme and O. Ritter, of the GeoForschungsZentrum Potsdam, section Deep Electromagnetic Sounding, for the motivation to built this facility, and H. Lühr for suggestions in designing the coil and for useful discussions.

\section{References}

[1] Kaufman, A.A. and Keller, G.V. Magnetotelluric Sounding Method Elsevier Scientific Puplishing Company 1981

[2] Vozoff, K., The magnetotelluric method, Electromagnetic

Methods in Applied Geophysics ed. M. N. Nabighian, Vol 3, p641-711, 1991

[3] METRONIX-Handboock

Broadbank Induction coil magnetometer MFS05

[4] Pulz, Eberhard and Ritter, Oliver, Entwicklung einer Kalibriereinrichtung für Induktionsspulenmagnetometer am GeoForschungs-Zentrum Potsdam

Scientific Technical Report STR01/10, Potsdam 2001

(http://www.gfz-potsdam.de/bib/pub/str0110/0110.htm. 